\title{
Analysis of the Contention Access Period (CAP) Model for the IEEE 802.15.7 Visible Light Communication
}

\author{
A. Musa, M. D. Baba, and M. A. Mansor
}

\begin{abstract}
IEEE 802.15.7 is introduced to standardize the visible light communication system for connecting devices via visible light spectrum. A slotted CSMA/CA MAC protocol defined in the standard to coordinate channel access for numbers of the optical wireless devices. In this paper, we proposed a Discrete Time Markov Chain (DTMC) model for modeling the behavior of the Contention Access Period (CAP) of the IEEE 802.15.7 to acquire valuable insightsand inner workings of the protocol. The through performance of the Contention Access Period (CAP) of the IEEE 802.15.7 is analyzed. ns-2 simulations are used to verify the accuracy of the proposed model. The results between the proposed model and ns-2 simulation are closely matched. Thus, verify the accuracy of the proposed model.
\end{abstract}

Index Terms-Discrete time Markov chain, IEEE 802.15.7, medium access layer, visible light communication.

\section{INTRODUCTION}

In recent years, interest in optical wireless as a favorable complementary technology for radio frequency (RF) communication has gained momentum supported by substantial deployments in solid state lighting technology and the released of the IEEE 802.15.7 draft standard [1]. The IEEE 802.15.7 compliant networks consist of two types of nodes, namely, a coordinator which initializes and manages the network and a remote device which communicate with each other via the coordinator. The IEEE 802.15.7 standard defines three types of topologies, namely, peer-to-peer, star, and broadcast as shown in Fig. 1. The IEEE 802.15.7 defines the first two layers of ISO/OSI stack protocol that includes Physical and Medium Access Control (MAC) layers. IEEE 802.15.7 MAC is a Carrier Sense Medium Access/Collision Avoidance (CSMA/CA) based protocol.

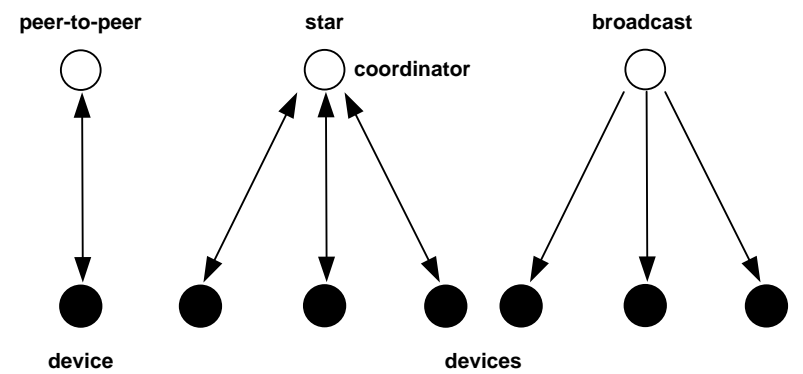

Fig. 1. IEEE 802.15.7 topologies defined in IEEE 802.15.7 draft standard.

Manuscript received January 20, 2013; revised April 20, 2013.

The authors are with Faculty of Electrical Engineering, UniversitiTeknologi MARA (UiTM), Shah Alam, Selangor, Malaysia (e-mail: anuarmusa@tmrnd.com.my).
There are several methods based on simulations and experiments that have been proposed for performance evaluation of CSMA/CA networks for the IEEE 802.11 [2], [3], IEEE 802.15.4 [4]-[6] and IEEE 802.15.7 [7]. However, it is importance to develop analytical tools that can predict accurately the network performance without resorting to a complicated analytical model, an extensive simulation or a tough experiment. An excellent and simple model for the IEEE 802.15.4 unsaturated traffic condition is presented in [8]. Specifically, a Discrete Time Markov Chain (DTMC) based model that described both the remote devices and the channels. In this paper, we extend [8] to cater for the IEEE 802.15.7 CSMA/CA MAC and include acknowledgement mechanism to make the analysis to closely conform to the IEEE 802.15.7 standard. The accurate model is import to understand and to gain valuable insight of the protocol.

The rest of this paper is organized as follows: Section II provides an overview of the IEEE 802.15.7 MAC; Section III describes the proposed MAC analytical model; simulation results are given in Section IV; and finally Section V concludes this paper.

\section{OVERVIEW OF THE IEEE 802.15.7}

The IEEE 802.15.7 describes two mechanisms for MAC channel access: contention based and contention free. Contention-based access allows devices to access the channel in contention access period (CAP) using a random access backoff algorithm. Contention-free access is managed by the coordinator through the use of guaranteed time slot (GTS) mechanism in contention free period (CFP) as shown in Fig. 2.

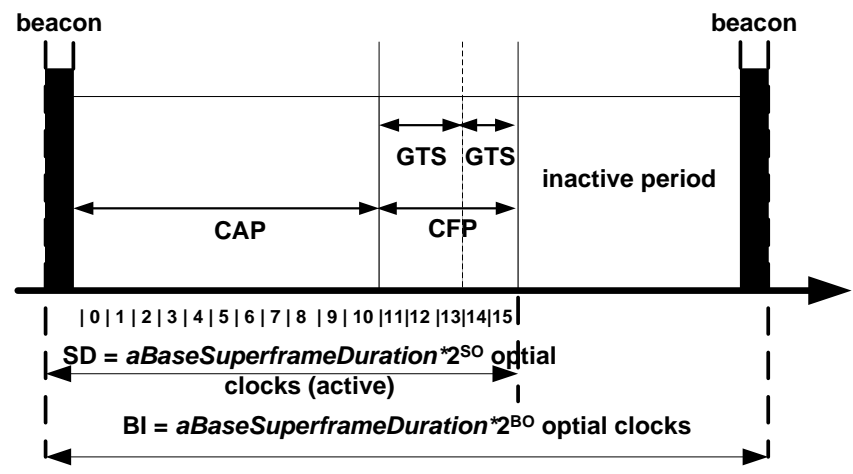

Fig. 2. IEEE 802.15.7 MAC in IEEE 802.15.7 draft standard.

In slotted CSMA/CA of the IEEE 802.15.7 as shown in Fig. 3, first the MAC sublayer initializes two variables: NB and BE. NB is the number of backoff times which the algorithm tries to backoff when the medium is busy. BE is the backoff exponent, which is related to the number of backoff periods a 
device has to wait before trying to access a channel. The default values of these parameters are listed in Table I. BE will be initialized with the value of $\operatorname{macMinBE}$. Based on BE value, the algorithm locates the boundary of the next backoff period for the device. The device generates a random number in the range of 0 to $\left(2^{\mathrm{BE}}-1\right)$ and then it waits until the random number is decreased to zero to perform clear channel assessment (CCA). The physical layer performs a CCA to check whether the channel is busy or idle. If the channel is sensed busy, the algorithm will increase the NB and BE values by one, with $\mathrm{BE}$ is equal or less than the value of macMaxBE. If NB exceeds the macMaxCSMABackoffs value, then the packet is discarded due to channel access failure. If the NB value is equal or less than macMaxCSMABackoffs value, the algorithm will relocate backoff period boundary. Then it will apply backoff delay process again. If the channel is idle, the packet is transmitted and the device waits for acknowledgment (ACK). The reception of ACK presents the successful transmission.

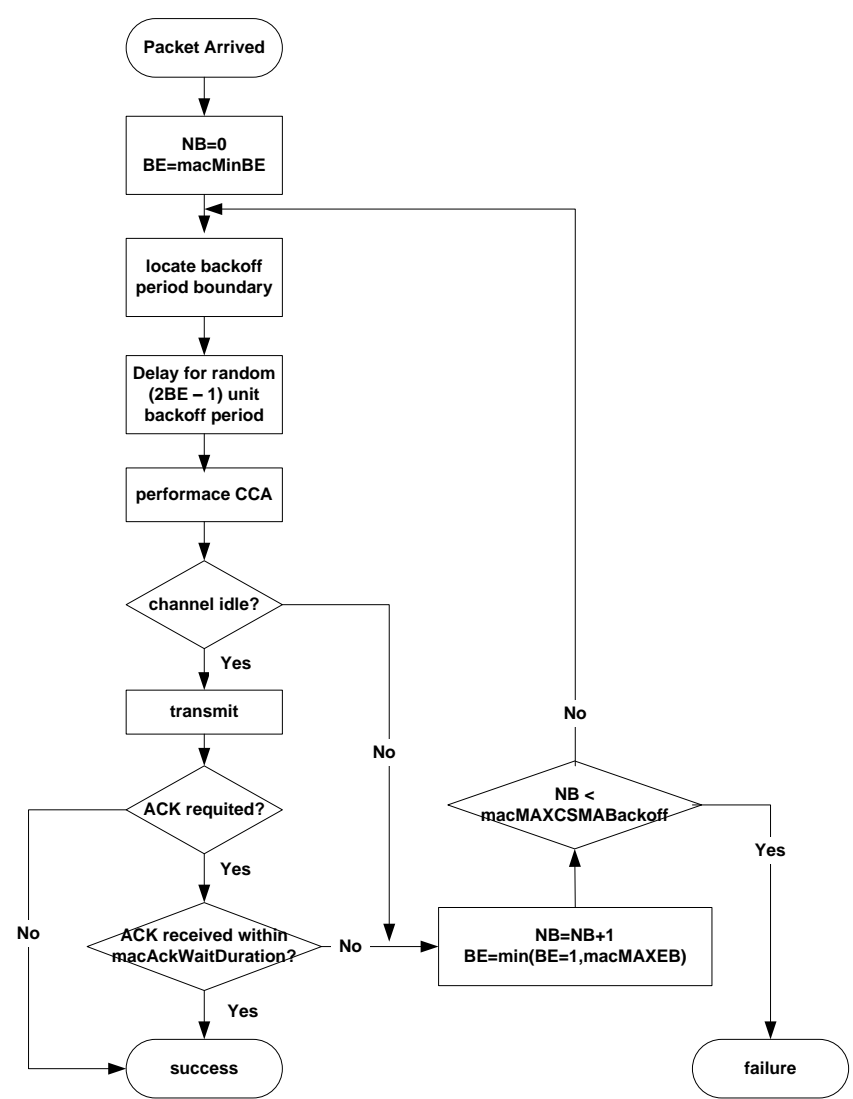

Fig. 3. IEEE 802.15.7 CSMA/CA algorithm for a slotted contention access period (CAP).

\section{ANALYTICAL MODEL}

\section{A. Model Assumptions}

The model presented will be based on the same assumptions as [8]. However, some modifications are made on these assumptions as well as the new assumptions are added due to the extension of the model. The following paragraphs summarize all of the assumptions.

Most of the IEEE 802.15.7 applications work under a one-hop star topology. There are fixed number $\mathrm{M}$ remote devices within the star topology, with a common coordinator where all nodes are within carrier sensing range of each other.
This is to ensure the transmission is not to be interrupted by other nodes (no hidden terminal).

Since the analysis is only concerned about the MAC performance in the CAP, it is assumed that superframe does not have a CFP and an inactive period. Therefore, the entire superframe duration is active; that means $\mathrm{SO}=\mathrm{BO}$.

In the acknowledged CAP analysis, the assumption is that there are no packet losses; the only cause of transmission failures is due to collisions. MAC level ACKs will always be sent after each successful data transmission and will always arrive to the destination node and no data packet retransmissions after a collision. In this scheme the coordinator notifies the remote node after a packet reception with an ACK. If the node does not receive an ACK, it assumes collisions have occurred; however, no retransmissions will be sent.

Data packets are assumed to be a fixed N-backoff slots duration and arrive at the nodes for transmission according to a Poisson arrival rate of $\lambda$ packets per packet duration. The probability $\mathrm{p}$ that a node will get a packet to transmit at the next slot is $p=\lambda / N$. In addition, no buffering is examined at the nodes. New packets are not accepted for transmission when the node is currently transmitting, or, is attempting a transmission.

\section{B. Model Analysis}

The behavior of the IEEE 802.15.7 system is modeled using a Discrete Time Markov Chain (DTMC) as shown on Fig. 4. Initially, the node is in the IDLE state until it receives a packet to be transmitted in a backoff slot, which occurs with probability $\mathrm{p}$. Then, it moves on to the state $\mathrm{BO}_{1}$ to perform the first backoff stage of the CSMA protocol. The node spends a random number of backoff slots chosen uniformly between 0 and $\left(2^{\mathrm{BE}}-1\right)$.

The node reaches the $\mathrm{CS} 1$ after leaving $\mathrm{BO}_{1}$, and to check that the channel is idle by performing the $\mathrm{CCA}$. If the channel is found idle, event characterized by probability $p_{i}^{c}$, the node would enter the TX state and start transmitting the packet.

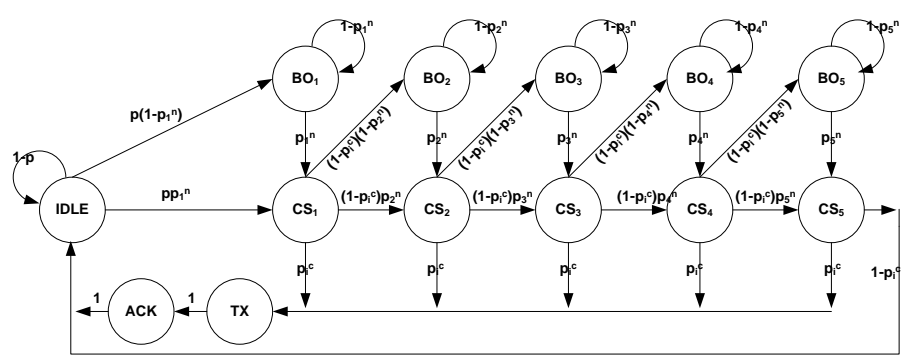

Fig. 4. DTMC node state model for slotted CSMA/CA. The notation BOi, $\leq i \leq 5$ and $\mathrm{CSj}, 1 \leq j \leq 5$ denotes the CCA attempt after the jth random backoff stage

If the channel is busy in the CCA attempt at state CS, which occurs with probability $\left(1-p_{i}^{c}\right.$, the node goes into the second backoff stage $\mathrm{BO}_{2}$, increasing the value of the $\mathrm{BE}$ by 1 , and repeats the same backoff and CCA procedure.

In general, the notation $\mathrm{CS}_{\mathrm{i}}, 1<i<5$ is to denote the $i^{\text {th }}$ random backoff stage, $\mathrm{BO}_{\mathrm{i}}$. When the node is in the TX state, it spends $N$ backoff slots in that state (since the length of a packet, in terms of number of backoff slots, is equal to $\mathrm{N}$ ) and then transitions to the ACK state where it waits to receive the acknowledgment from the coordinator. From this ACK, the node finally goes back to the initial IDLE state with 
probability 1 .

The complete Markov chain for the remote node is constructed starting in the IDLE state, and moving all the way around through the chain until either the ACK packet has been correctly received, the MacAckWaitDuration is consumed without receiving the ACK, or until the maximum number of backoff stages macMaxCSMABackoff is reached and access channel failure is asserted.

The steady state occupancy of this chain can be obtained by solving the Markov equation system in (1). The notation $\pi\left(\right.$ state $\left._{\mathrm{i}}\right)$ denotes the long term proportion of transitions into state $_{\mathrm{i}}$

$$
\begin{gathered}
\pi(\text { idle })=(1-p) \pi(i d l e)+\left(1-p_{i}^{c}\right)\left(C S_{5}\right) \\
+\pi(A C K) \pi\left(B O_{1}\right) \\
=\left(1-p_{1}^{n}\right) \pi\left(B O_{1}\right)+p\left(1-p_{1}^{n}\right) \pi(i d l e) \\
\pi\left(B O_{2}\right)=\left(1-p_{2}^{n}\right) \pi\left(B O_{2}\right)+\left(1-p_{2}^{n}\right)\left(1-p_{i}^{c}\right) \pi\left(C S_{1}\right) \\
\pi\left(B O_{3}\right)=\left(1-p_{3}^{n}\right) \pi\left(B O_{3}\right)+\left(1-p_{3}^{n}\right)\left(1-p_{i}^{c}\right) \pi\left(C S_{2}\right) \\
\pi\left(B O_{4}\right)=\left(1-p_{4}^{n}\right) \pi\left(B O_{4}\right)+\left(1-p_{4}^{n}\right)\left(1-p_{i}^{c}\right) \pi\left(C S_{3}\right) \\
\pi\left(B O_{5}\right)=\left(1-p_{5}^{n}\right) \pi\left(B O_{5}\right)+\left(1-p_{5}^{n}\right)\left(1-p_{i}^{c}\right) \pi\left(C S_{4}\right) \\
\pi\left(C S_{1}\right)=p\left(p_{1}^{n}\right) \pi(i d l e)+\left(p_{1}^{n}\right) \pi\left(B O_{1}\right) \\
\pi\left(C S_{2}\right)=\left(1-p_{i}^{c}\right)\left(p_{2}^{n}\right) \pi\left(C S_{1}\right)+\left(p_{2}^{n}\right) \pi\left(B O_{2}\right) \\
\pi\left(C S_{3}\right)=\left(1-p_{i}^{c}\right)\left(p_{3}^{n}\right) \pi\left(C S_{2}\right)+\left(p_{3}^{n}\right) \pi\left(B O_{3}\right) \\
\pi\left(C S_{4}\right)=\left(1-p_{i}^{c}\right)\left(p_{4}^{n}\right) \pi\left(C S_{3}\right)+\left(p_{4}^{n}\right) \pi\left(B O_{4}\right) \\
\pi\left(C S_{5}\right)=\left(1-p_{i}^{c}\right)\left(p_{5}^{n}\right) \pi\left(C S_{4}\right)+\left(p_{5}^{n}\right) \pi\left(B O_{5}\right) \\
\pi(T x)=\pi(A c k) \\
\pi(i d l e)+\pi(T x)+\pi(A c k)+\sum_{i=1}^{5}\left[C S_{i}+B O_{i}\right]=1
\end{gathered}
$$

The probability that a node starts transmission at any generic backoff slot, or equivalently, the steady state probability that node transmits, can be shown by:

$$
\begin{aligned}
& p_{t}^{n}=p_{c s}^{n} p_{i}^{c} \\
& =\left(\frac{\sum_{i=1}^{5} \pi\left(C S_{i}\right)}{\pi(\text { idle })+\pi(T x)+\pi(A c k)+\sum_{i=1}^{5}\left[\pi\left(C S_{i}\right)+\pi\left(B O_{i}\right)\right]}\right) p_{i}^{c}
\end{aligned}
$$

Knowing the probability $p_{t}^{n}$ that an individual station transmits in a generic slot $N$, a Markov chain model for the channel states can be developed. The channel state diagram is shown in Fig. 5.

The channel is in the Idle state, when there is no transmission. It remains in the Idlestate, if no node transmitting, which occurs with probability equal to $\propto=\left(1-p_{t \mid i}^{n}\right)^{M}$, where $\mathrm{M}$ is the number of remote nodes, excluding the coordinator. The probability that any node begins transmission, given that the channel is idle is equal to $p_{t}^{n} \quad(3.2)$.

If only one node is transmitting and other nodes remain idle, then probability equal to $\beta=M p_{t \mid i}^{n}\left(1-p_{t \mid i}^{n}\right)^{M-1}$. Then channel transitions to the Success state. If more than one node starts to transmit at thesame time, the channel goes to the Failure state, indicating an unsuccessful transmission due to collision; this happen at probability of $\delta=1-\propto-\beta$. It spends $\mathrm{N}$ slots each inthe Success and Failure states and then returns to the Idlestate with probability 1 .

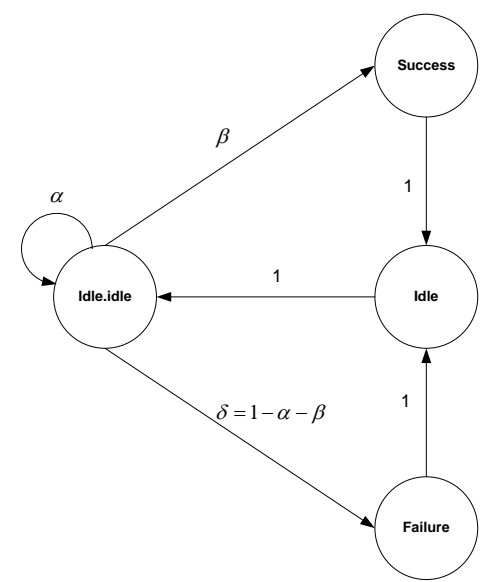

Fig. 5. DTMC channel state model. The transition probability of $\propto$ and $\beta$ are given by $\propto=\left(1-p_{t \mid i}^{n}\right)^{M}, \beta=M p_{t \mid i}^{n}\left(1-p_{t \mid i}^{n}\right)^{M-1}$, respectively. $\mathrm{M}$ is the number of the mobile stations excluding the coordinator.

The notation $\pi\left(\right.$ state $\left._{i}\right)$ denotes the long term proportion of transitions into state ${ }_{\mathrm{i}}$ as shown below.

$$
\begin{gathered}
\pi_{i}^{c}=\propto \pi_{i}^{c}+\pi_{b}^{c} \\
\pi_{s}^{c}=\beta \pi_{i}^{c} \\
\pi_{f}^{c}=(1-\propto-\beta) \pi_{i}^{c} \\
\pi_{f}^{c}+\pi_{i}^{c}+\pi_{b}^{c}+\pi_{s}^{c}=1
\end{gathered}
$$

Solving, equations (3) yields the following steady state solution as shown in (4):

$$
\begin{gathered}
\pi_{i}^{c}=1 /(3-2 \alpha) \\
\pi_{s}^{c}=\beta /(3-2 \alpha) \\
\pi_{f}^{c}=(1-\alpha-\beta) /(3-2 \alpha) \\
\pi_{b}^{c}=(1-\propto) /(3-2 \alpha)
\end{gathered}
$$

The probability that the channel is idles, $p_{i}^{c}$, can be obtained by calculating the idle time spent in each state the steady state probability given by:

$$
\begin{aligned}
p_{i}^{c} & =\pi_{i}^{c} /\left(\pi_{i}^{c}+N \pi_{s}^{c}+N \pi_{f}^{c}+\pi_{b}^{c}\right) \\
& =1 /(1+N \beta+N(1-\alpha-\beta)+(1-\alpha)) \\
& =1 /(1+N(1-\alpha)+(1-\alpha)) \\
& =1 /(1+(N+1)(1-\alpha)) \\
& =1 /\left(1+(N+1)\left(1-\left(1-p_{t \mid i}^{n}\right)^{M}\right)\right)
\end{aligned}
$$

$p_{t \mid i}^{n}$ is the probability that the node transmit given that the channel is idle in the previous backoff slot, which is the probability of the node sensed the channel in that backoff slot, $\mathrm{p}_{\mathrm{cs}}^{\mathrm{n}}$. Since, $p_{t \mid i}^{n}$ is a function of $p_{i}^{c}$ and $p_{i}^{c}$ in is a function of $p_{t \mid i}^{n} \quad$ (5), we have a consistent set of equations which can be solved numerically. Analytically, these equations are solved using Matlab [9].

\section{Aggregate Throughput}

The aggregate channel throughput can be derived from the Markov chain steady state probability of being in the Success state of Fig. 5. The fraction of time spend in each state can be obtained by calculating the actual time spent in each state. 
Observing that the time spends $\mathrm{N}$ backoff slots in the Successor Failure states and 1 backoff slot in each of the other states, thus, the throughput can be obtained as follows:

$$
\begin{gathered}
S=N \pi_{s}^{c} /\left(\pi_{i}^{c}+N \pi_{s}^{c}+N \pi_{f}^{c}+\pi_{b}^{c}\right) \\
=N \beta /(1+N \beta+N(1-\alpha-\beta)+(1-\alpha)) \\
=N \beta /(1+N(1-\alpha)+(1-\alpha))(6) \\
=N \beta /(1+(N+1)(1-\alpha)) \\
=N M p_{t \mid i}^{n} \quad\left(1-p_{t \mid i}^{n}\right)^{M-1} /\left(1+(N+1)\left(1-\left(1-p_{t \mid i}^{n}\right)^{M}\right)\right)
\end{gathered}
$$

\section{RESULTS}

The analysis and simulations presented in this paper are based on the network that is configured as Table I. Extensive ns-2[10] simulations have been conducted based on the CSMA/CA algorithms in the CAP and verify the theoretical analysis.

TABLE I: MAC CONFIGURATIONS

\begin{tabular}{ll}
\hline \hline symbol & value \\
\hline Data Rate & $250 \mathrm{kbps}$ \\
aTurnaroundTime & 8 optical clock \\
CCA & 8 optical clock \\
aUnitBackoffPeriod & 20 optical clock \\
SIFS & 12 optical clock \\
LIFS & 40 optical clock \\
aMaxSIFSFrameSize & 18 optical clock \\
macRespondWaitTime & 32 optical clock \\
ACK Transmission & 22 optical clock \\
macMinBE & 3 \\
macMaxCSMABackoffs & 5 \\
Frame Payload & 127 bytes \\
aMaxPHYFrameSize & 1023 \\
\hline \hline
\end{tabular}

Fig. 6 shows that the numerical analysis matches well the simulation results; thus, validate that analysis model is accurate.

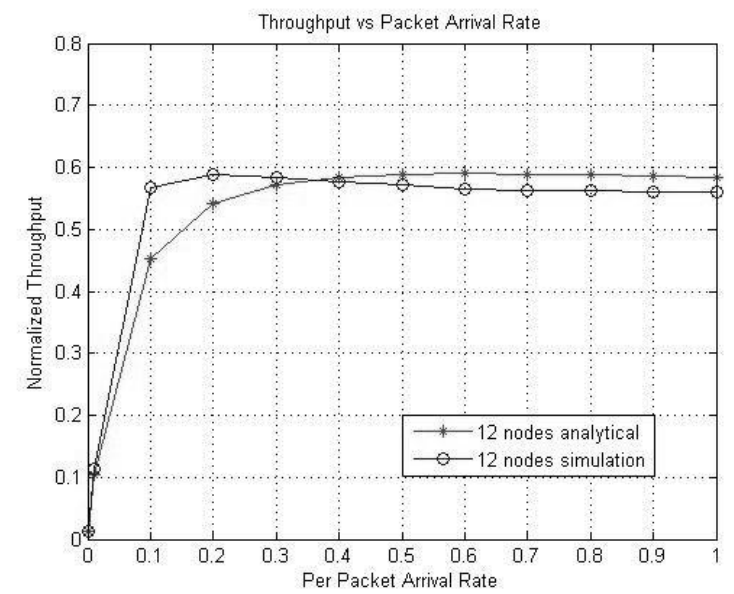

Fig. 6. Channel throughput as a function of packet arrival rate, $\lambda$. Number of mobile station, $\mathrm{M}$ is 12 and packet length $\mathrm{N}$ is 10 backoff slots.

\section{CONCLUSION}

In this paper, we have presented the analysis on the modelling of the CAP channel access mechanism for IEEE802.15.7 protocol based on the Discrete Time Markov
Chain (DTMC) state node model. $n s-2$ simulations are used to verify the accuracy of the proposed model. From the numerical results, it indicates that the proposed CAP state node model match closely to the $n s-2$ simulation result.Thus, verify the accuracy of the proposed model.

\section{REFERENCES}

[1] Wireless Medium Access Control (MAC) and Physical Layer (PHY) specifications, IEEE 802.15.7-2011 draft8 standard.

[2] G. Bianchi,"Performance analysis of the IEEE 802.11 distributed coordination function," IEEE Journal on selected areas in communications, vol. 18, pp. 535-547, 2000.

[3] J. Yin, "The Analysis of Performance of IEEE 802.11 MAC Protocol Using Markov Chain," IJCSNS International Journal of Computer Science and Network Security, vol. 7, no. 12, pp. 27-37, December 2007.

[4] P. Park, P. Di Marco, P. Soldati, C. Fischione, and K. H. Johansson, “A generalized Markov chain model for effective analysis of slotted IEEE 802.15.4," in Proc. International Conference on Mobile Adhoc and Sensor Systems, pp. 130-139, October 2009.

[5] H. Wen, C. Lin, and Z. J. Chen et al., "An improved Markov model for IEEE 802.15.4 slotted CSMA/CA mechanism," Journal of computer science and technology, vol. 24, pp. 495-504, May 2009.

[6] F. Wang, D. Li, and Y. Zhao, "Analysis of CSMA/CA in IEEE802.15.4," IET Communications, pp. 2187-2195, 2011.

[7] V. V. Huynh, L. M. Tuan, and Y. M. Jang, "Priority MAC Based on Multi-parameter for IEEE 802.15.7 VLC," pp. 257-260, ICTC, 2011.

[8] I. Ramachandran, A. K. Das, and S. Roy, "Analysis of the contention access period of IEEE 802.15.4 MAC,"ACM New York, 2007.

[9] Matlab Website. [Online]. Available: http://www.matworks.com.

[10] USC Information Sciences Institute. Marina del Rey, CA. Network Simulator ns-2. [Online]. Available: http://www.isi.edu/nsnam/ns

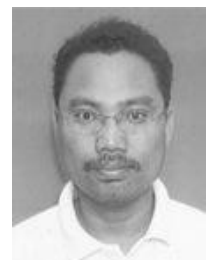

A. Musa received a B.Sc. degree in electrical engineering and computer science from Washington University, St. Louis, MO., USA in 1994. He completed his M. Sc. in computer science from Vanderbilt University, Nashville, TN., USA in 1997. $\mathrm{He}$ is currently a Ph.D. student at the Faculty of Electrical Engineering, UniversitiTeknologi MARA (UiTM), Malaysia.His main research interestsarein the area of wireless communication and embedded system. He is a senior member of the IEEE association.

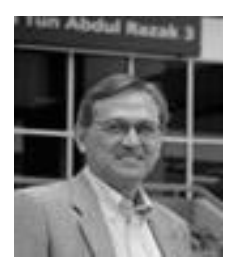

M. D. Baba received his $\mathrm{PhD}$ from University of Sussex, UK in 1996. Currently he is a Professor in Computer and Communication Engineering at the Centre for Computer Engineering Studies, Faculty of Electrical Engineering, UniversitiTeknologi MARA (UiTM), Malaysia. His research interests include computer networking, cloud computing, wireless networking, wireless sensor networks, cognitive networks and mobile communication.

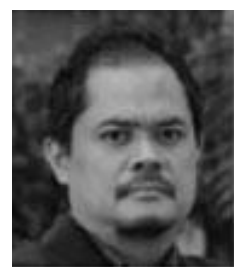

M. A. Mansor received his $\mathrm{PhD}$ from University of Sheffield, UK in 1998. Currently he is an associate professor at the Centre for Computer Engineering Studies, Faculty of Electrical Engineering, UniversitiTeknologi MARA (UiTM), Malaysia. His research interests include computer networking, computer engineering, cloud computing, wireless networking, robotics and AI. 